\title{
Utilidad clínica del conteo de células precursoras hematopoyéticas (CPH) comparado con el recuento de células CD34+ en sangre periférica (SP) para la predicción de la realización de aféresis
}

- Claudia Sossa Melo, Carlos Pérez Martínez, Sara Inés Jiménez S., Ángela Peña C., Luis A. Salazar M., Miguel Ochoa, Héctor Meléndez

Fundación Oftalmológica de Santander, Clínica Carlos Ardila Lulle (Foscal), Universidad Autónoma de Bucaramanga (UNAB), Bucaramanga (Santander).

Contacto: claudiasossa@gmail.com

Introducción y objetivos. El éxito de un trasplante de precursores hematopoyéticos de SP depende de un adecuado número de células CD34+ infundidas. El momento óptimo de la colecta por aféresis es clave. El conteo de células CD34+ en SP $\geq 10 \times 106 /$ es utilizado como criterio para la realización de aféresis. Las CPH en SP se correlacionan con las células CD34+ en SP, siendo una alternativa rápida (90 segundos), sin operador dependiente, económica (USD9,8), comparado con CD34+ de SP, que es costosa (USD110,34) y consume mayor tiempo. Nuestro objetivo fue evaluar la utilidad clínica del conteo de $\mathrm{CPH}$ en SP tomando como referencia un conteo de CD34+ en $S P \geq 10 \times 106 / 1$.

Materiales y métodos. Evaluación de la prueba diagnóstica en 109 determinaciones de 60 pacientes con indicación de trasplante de progenitores hematopoyéticos de SP en la Clínica Carlos Ardila Lulle.

Resultados. El análisis mostró que el punto de corte de $26 \mathrm{CPH} / \mathrm{ul}$ en SP presenta su máxima capacidad discriminatoria para correlacionarse con un valor de CD34+ en SP $\geq 10 \times 106 /$, con una sensibilidad del $78,5 \%$, especificidad del $85 \%$, valor predictivo positivo del $95 \%$, negativo del $95 \%$ y un área bajo la curva de 0,87 , prediciendo correctamente el valor de CD34+ en SP $\geq 10 \times 106 /$ en el $79,8 \%$ de todas la mediciones.

Conclusiones. La determinación de CPH en SP es una alternativa útil dada su capacidad discriminatoria, el rápido acceso y la disminución de costos.

\section{Trasplante autólogo en niños. Experiencia Fundación Homi}

\section{Marcela Estupiñán Peñaloza, Mauricio Chaparro Alzogaray}

Fundación Hospital de la Misericordia, Bogotá, D.C.

Contacto: mestupinanp@fundacionhomi.org.co

Introducción y objetivos. El trasplante autólogo permite el rescate de la hematopoyesis luego de dosis altas de quimioterapia. Es especialmente útil en tumores sólidos y enfermedades autoinmunes. El trasplante autólogo es una alternativa en algunos pacientes con enfermedades extensas de alto riesgo o recurrentes en el caso de neoplasias, y en caso de refractariedad a terapias previas en esclerosis sistémica progresiva.

Materiales y métodos. Entre agosto de 2007 y mayo de 2015 se han realizado 43 trasplantes autólogos. Las principales indicaciones fueron neuroblastoma, enfermedad de Hodgkin, linfoma no Hodgkin,
TNEP, leucemia promielocítica y un paciente con esclerosis sistémica progresiva. Los regímenes de acondicionamiento fueron para neuroblastoma CEM o Bumel, linfoma de Hodgkin BEAM o Bumel, TNEP Bumel, linfoma no Hodgkin BUCY. En el paciente con esclerosis sistémica progresiva, el acondicionamiento fue ciclofosfamida-ATG.

Resultados. La sobrevida global y sobrevida libre de enfermedad en esta cohorte fue del $65 \%$. No hubo muertes relacionadas con la terapia (MRT 0\%).

Conclusiones. El trasplante autólogo es una terapia segura con mortalidad relacionada con trasplante del $0 \%$ en nuestra serie. 